\title{
Effect of docosahexaenoic acid on axonal damage after spinal cord injury*
}

Rachael E. Ward,

Wenlong Huang,

Jodie C. E. Hall,

John V. Priestley

Adina T. Michael-Titus

\section{Neuroscience Centre,}

Institute of Cell \& Molecular Sciences,

Bart's and the London School of Medicine and Dentistry,

Queen Mary University of London,

4, Newark Street,

London E1 2AT

We have shown that the omega-3 polyunsaturated fatty acid docosahexaenoic acid (DHA) increases neuronal and oligodendrocyte survival in a rat compression model of spinal cord injury [1]. As one of the major factors that contribute to the loss of neurological function following spinal cord injury is axonal pathology, in the present study we have focused on the effects of DHA on axonal and dendritic damage after compression injury. A laminectomy was performed in adult female Wistar rats (230-250 g) at thoracic level T12 and a $50 \mathrm{~g}$ weight was applied to the dorsal surface of the dura mater for $5 \mathrm{~min}$. $30 \mathrm{~min}$ following compression, rats were intravenously given either saline or DHA (250 nmol/kg). After the DHA injection, one group of rats was placed on a DHA-enriched diet (400 mg/kg/day) whilst one group received normal chow. 5 animals were used per group. 1 or 6 weeks post-injury, animals were perfused intracardially with saline and $4 \%$ paraformaldehyde. $15 \mu \mathrm{m}$ spinal cord sections were then processed for immunohistofluorescence using the following primary antisera: $\beta$-amyloid precursor protein ( $\beta$-APP) (1:500, Zymed), neurofilament-200 (1:2000, Sigma), SMI32 (non-phosphorylated neurofilament, 1:500, Sternberger Monoclonals Inc.), microtubule associated protein2 (MAP-2) (1:500, Sigma) and myelin basic protein (MBP) (1:500, Roche). Data was expressed as means \pm SEM and analysed with one-way ANOVA and post-hoc pairwise comparisons using the Bonferroni test. $\beta$-APP-positive axons were scarce in non-injured animals but prominent in the white matter tracts of saline-treated injured animals. At 1 week, in both groups of DHA-treated animals, $\beta$-APPpositive axon number in the dorsal columns was significantly reduced vs. saline-treated injured animals (saline

$76 \pm 10$, DHA injection $34 \pm 9$, DHA injection and diet $33 \pm 11 ; \mathrm{p}<0.05)$. By 6 weeks, $\beta$-APP immunoreactivity in the corticospinal tract and ventrolateral white matter was also significantly reduced vs. saline in both DHA-treated groups $(p<0.05)$. The number of non-phosphorylated neurofilament-positive axons was reduced in injured animals compared to controls. At 1 week, this reduction was significantly reversed in the two DHA-treated groups in the ventrolateral white matter (control $23 \pm 2$, saline $11 \pm 1$, DHA injection $19 \pm 3$, DHA injection and diet $17 \pm 1$; $p<0.05)$. By 6 weeks, DHA treatment also significantly protected against the loss of SMI32 in the dorsal columns and ventral white matter $(p<0.05)$. The effect of both DHA treatments on the reduction in the number of myelin rings and myelinated neurofilament-positive axons that occurs 1 week post-injury was heterogeneous and requires further evaluation. At 1 week post-injury, neither DHA treatment protected against the injury-induced loss of dendritic MAP-2. By 6 weeks, DHA injection and diet this loss was significantly reduced in the dorsalateral white matter (saline -95\%, DHA injection -74\%, DHA injection and diet $-45 \% ; \mathrm{p}<0.05)$. Both DHA treatments significantly prevented loss of cell body MAP-2 in the dorsal horn by 1 week post-injury (saline $-76 \%$, DHA injection $-47 \%$, DHA injection and diet $-30 \% ; p<0.05)$ and ventral horn $(p<0.05)$. These results indicate that DHA has major neuroprotective effects against the axonal and dendritic damage caused by spinal cord injury.

\section{Reference}

1. King VR, et al. J Neurosci 2006; 26: 4672-80.

* This work was supported by the Corporate Action Trust.

\section{POSTERS}

